\title{
Posterior Interosseous Artery Flap for Hand Defects
}

\author{
Brig BB Dogra*, Col Manmohan Singh ${ }^{+}$, Lt Col B Chakravarty", Lt Col S Basu**
}

\begin{abstract}
Background : Reconstruction of soft tissue defects in the hand need an early, single stage and well vascularised cover to achieve the best functional result. Usually a full thickness graft is required since vital structures like tendons, bones and joints are exposed and often there is need for secondary reconstruction.

Methods: We managed 12 cases of complex defects over the hand in the last 2 years with the posterior interosseous artery flap. Results: In 5 cases the defect was due to blast injury and in 4 because of crush injury. Males predominated in the ratio of 5:1. The defect was most often in the $1^{\text {st }}$ web space and the largest flap was $11 \times 8 \mathrm{~cm}$. In all but one case the donor site was covered by split skin graft, which settled well. 2 patients had superficial flap necrosis needing debridement and skin graft.

Conclusion : Flap based on reverse flow in the posterior interosseous artery is a versatile and reliable source for full thickness cover of complex soft tissue defects in the hand.
\end{abstract}

MJAFI 2006; 62 : 33-35

Key Words: Soft tissue defect of hand; Posterior interosseous artery flap.

\section{Introduction}

$\mathrm{R}^{\mathrm{s}}$ econstruction of soft tissue defects of the hand requires early cover by a single stage procedure to minimize infection, allow early mobilisation and reduce hospital stay and thus achieve good functional result. If local flaps are insufficient to meet the requirement then regional flaps need to be used. A septocutaneous island flap based on the posterior interosseous artery raised from the posterior aspect of forearm originally described by Zancolli and Angrigiani in 1985 [1] has wide application in reconstruction of soft tissue defects on the dorsum of hand, wrist and first web space [2]. This flap has the advantage over other available flaps in that it does not require sacrifice of any vessel essential for perfusion of hand. We present our experience in reconstruction of 12 cases of hand defects using the

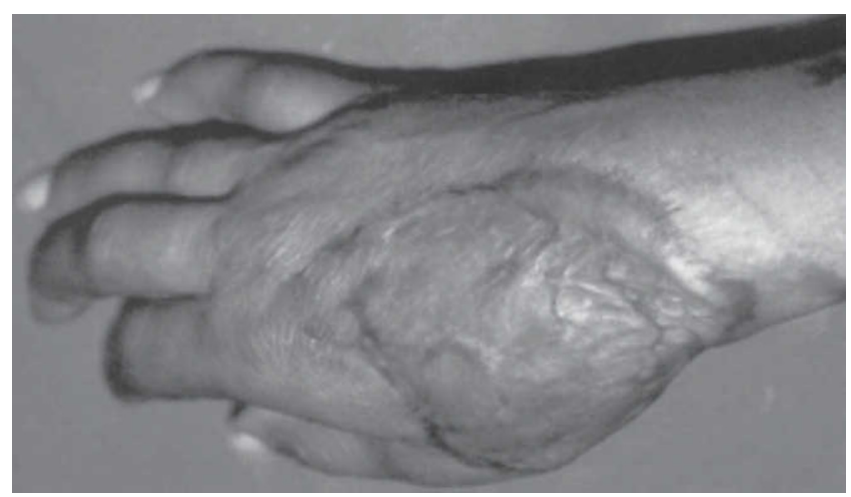

Fig. 1: A case of crush injury dorsum of hand and wrist managed initially by skin grafting for flap cover. posterior interosseous artery flap.

\section{Material and Methods}

Over the past 2 years, 12 cases of soft tissue defects of the hand underwent reconstruction using posterior interosseous artery flap. They comprised 5 cases of blast injury, 4 cases of crush injury (Fig 1), 2 cases of post burn defect and 1 case due to extravasation of anticarcinogenic drug (Fig 2). Of the 12, 10 were males with age ranging between 15 and 38 years and 2 were females aged 30 and 65 years. The skin flap size varied from $6 \times 5 \mathrm{~cm}$ to $11 \times 8 \mathrm{~cm}$. In 11 cases the donor site defects required skin grafting and 1 case was directly closed.

We have followed the operative technique as described by H.Costa et al $[3,4]$. Patient is placed supine and surface marking of the posterior interosseous artery is drawn along a line joining the lateral epicondyle of humerus and the ulnar styloid with the forearm in full pronation. A point $9 \mathrm{~cm}$ distal to the lateral epoicondyle of humerus marks the center of the

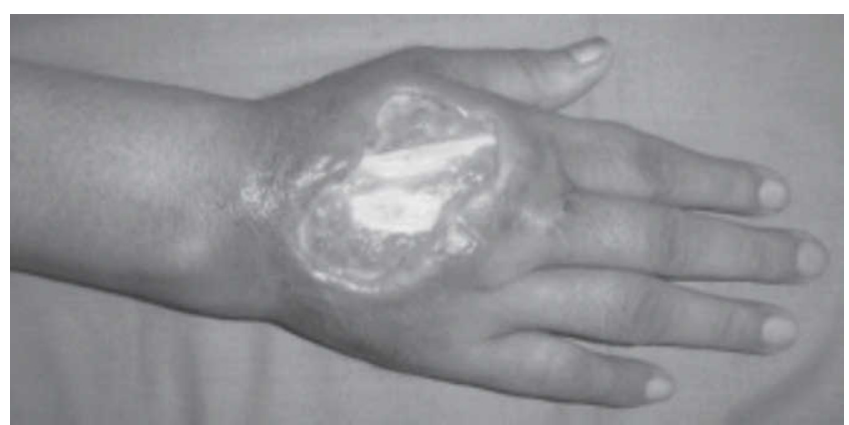

Fig. 2: Defect over the dorsum of hand due to extravasation of anti-carcinogenic drug with exposed extensor tendons.

*DDMS, HO Bengal Area (Med), Fort William, Kolkata, \#**Classified Specialist(Surg \& Reconstructive Surg), CH(SC), Pune-40, +Senior Adv (Surg), CH(SC), Kolkata.

Received : 24.03.2004; Accepted : 14.10.2004 


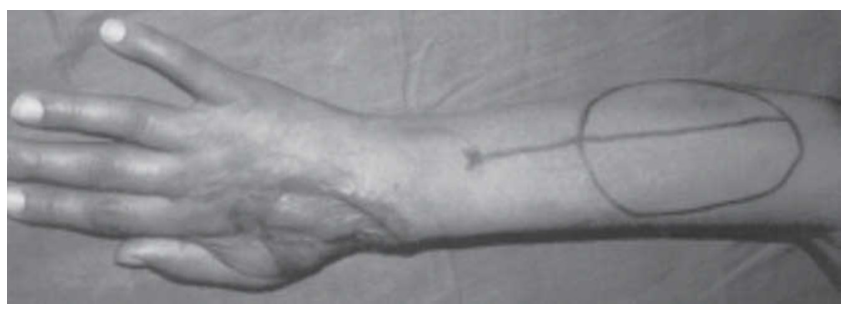

Fig. 3 : Posterior interosseous artery flap marking. ' $\mathrm{X}$ ' denotes the Pivot point of the flap.

fascio cutaneous element of the flap. The important anatomical consideration is the fascial septum between the extensor carpi ulnaris and extensor digiti minimi muscles, in which the vessels lie oriented sagitally. In the distal half, the artery is relatively superficial but in the proximal half the septum lies underneath the extensor digiti-minimi and so the vessel is more deeply situated. This is the main reason why dissection must be performed from distal to proximal. The point of anastomosis between posterior interosseous artery and anterior interosseous artery is marked $2 \mathrm{~cm}$ proximal to the radiocarpal joint and is the pivot point of the flap. This marking determines the most distal point about which the arc of rotation is taken although the centre of rotation may be more proximally located if desired [2] (Fig 3). The flap is tunnelled to the recipient area from its pivot point. Donor area can be directly closed if the flap is less than $5 \mathrm{~cm}$ but requires a split skin graft if the width is more.

\section{Results}

The flap settled nicely over the recipient area in 10 cases (Figs. 4 \& 5). In one there was superficial epidermal loss and in another there was partial flap necrosis. In both cases, the flaps settled subsequently with conservative management.

\section{Discussion}

For reconstruction of hand defects with substantial skin loss single stage procedure that can provide well vascularised flap coverage at the earliest offers the best possible functional results with reduction in fibrosis, scarring and hospital stay. The distally based radial artery forearm flap is considered the workhorse for covering large hand defects. Its advantages are a large skin paddle with reliable reversed perfusion [2]. But this flap should not be used in badly mutilated hands where the additional loss of the radial artery could compromise hand viability [5], though some authors have recommended that the flap can be raised in such cases if the pivot point is kept $5 \mathrm{~cm}$ proximal to the wrist crease [6]. The ulnar artery based flap is based on the major arterial supply to the hand and sacrificing the ulnar artery is the major disadvantage of this flap. The dorso ulnar flap, based on the ascending branch of the dorso ulnar artery is a distally based flap but has a short pedicle with limited rotation. Only defects on the ulnar-dorsal side of the hand and proximal palm can be reached [3]. The anterior interosseous flap with its skin paddle on the dorso radial

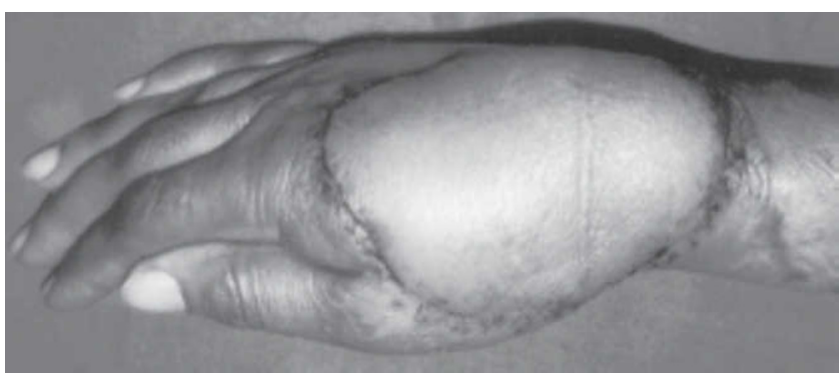

Fig. 4 : Post-operative result in the case of crush injury hand depicted in Fig. 1.

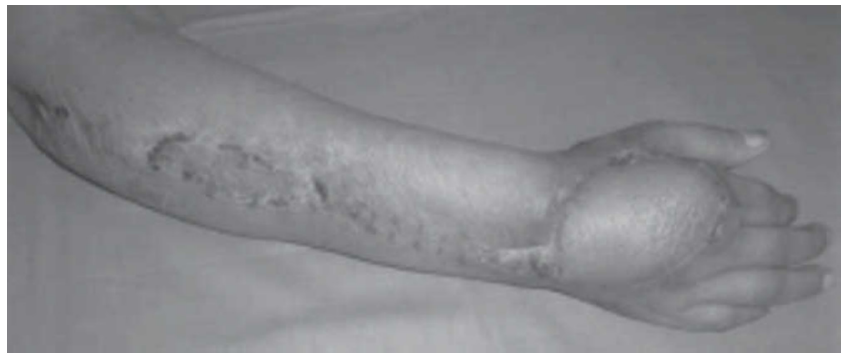

Fig. 5 : Post-operative result in the case of post anti-carcinogen extravasation hand defect depicted in Fig. 2.

side can provide skin, tendon, muscle and bone of distal radius but dissection is demanding involving fragile and anatomically variable vessels[3]. Moderate to large cutaneous defects of hand require distant flaps for reconstruction. Reverse volar forearm flap provides a good amount of tissue for coverage. However, a main source of the blood supply to the hand is disrupted and frequently an additional venous anastomosis is recommended to prevent edema and to facilitate drainage[2].

The posterior interosseous vessels offer a reliable blood supply to the skin of the posterior aspect of forearm and sacrifice of this vessel is not detrimental to hand vascularity. Another advantage is the anatomical basis of the retrograde flow of this flap by the anastomosis between the posterior and anterior interosseous arteries and so even with damage of radial or ulnar arteries or palmar arches this flap can still be used [4]. The distally pedicled posterior interosseous artery flap is suitable for providing vascular skin to the dorsal region of hand as far as the proximal phalanx of the thumb and the first web space [7]. It can also be raised as an osteofasciocutaneous unit, including a vascularised bone segment from the ulna for thumb reconstruction [4]. The flap can be based proximally to repair defects in the elbow region with direct flow through the posterior interosseous artery. An advantage of this flap compared to other forearm flaps is that being based on the dorsal aspect of forearm it does not interfere with the lymphatic drainage of the hand as seen in other forearm flaps (Radial or ulnar) which are based volarly [2].

In our series we found that in one case the main vessel of the posterior interosseous artery dipped below 
the extensor digiti minimi in the mid forearm level instead of running in septum between the extensor carpi ulnaris and extensor digiti minimi and then continued proximally in the septum between extensor digiti minimi and extensor digitorum communis. Therefore harvesting of the flap required removal of a fringe of extensor digit muscle along with the vessel. In another case, we found that the main vessel and the major perforator in midforearm were of extremely small calibre requiring meticulous dissection and in the same case the flap underwent partial necrosis. Similar variations in the vasularity have been documented by several authors [7,8], who have recommended additional preoperative Doppler examination of the perforating branches of the interosseous arteries in the proximal and middle third of forearm to facilitate raising an alternative flap distally pedicled either on the posterior or anterior interosseous artery. However there are other authors who have not found any gross anatomical variation in their patients [9]. The flap is therefore extremely reliable as demonstrated by several authors $[2,3,10]$.

\section{References}

1. Claudio Angrigiani, Daniel Grilli, Daniel Dominikow, Eduardo A Zancolli. Posterior interosseous reverse forearm flap: experience with 80 consecutive cases. Plast Reconst Surg 1993;
92;2: 285-93.

2. Cormack GC, Lamberty BGH. The arterial anatomy of skin flaps, Churchill Livingstone, $2^{\text {nd }}$ ed. 1994; 407-9.

3. Costa H, Gracia ML, Vranchx J, Cunha C, Conde A, Soutar D. The posterior interosseous flap: A review of 81 clinical cases and 100 anatomical dissections - assessment of its indications in reconstruction of hand defects. Br J Plastic Surgery. 2001; 54: 28-33.

4. Costa H, Comba H, Martins A, Rodrigues J, Reis J, Amaranti J. Further experience with the posterior interosseous artery flap. Br J Plastic Surgery 1991; 44: 449-55.

5. Cormack GC, Duncan MJ, Lamberty BGH. The blood supply of the bone component of the compound osteocutaneous radial artery forearm flap: an anatomical study. Br J Plastic Surgery. 1986; 39: 173-5.

6. Naasan A, Quaba AA. Successful transfer of two reverse forearm flaps despite disruption of both palmer arches. Br J Plastic Surgery. 1990; 43: 476-9.

7. Giunta R, Lucas B. Impossible harvest of the posterior interosseous artery flap: a report of an individalised salvage procedure. Br J Plastic Surgery. 1998; 51: 642-5.

8. Costa H, Soutar DS. The distally based island posterior interosseous artery flap. Br J Plastic Surgery. 1988; 41: 221-7.

9. Balakrishnan G, Kumar BS, Hussain SA. Reverse flow posterior interosseous artery flap revisited. Plast Reconstr Surg 2003; 111: 2364-69.

10. Koch H, Kursomovic, Hubmer M. Defects on the dorsum of the hand- the posterior interosseous artery flap and it's alternative. Hand Surgery. 2003; 108: 205-12.

\section{ATTENTION SUBSCRIBERS}

Subscription rates for MJAFI are :-

(i) Serving AMC/AD Corps officers (through AFMS $(\mathrm{O})$ )

(ii) Life Membership (for retiring officers)

(iii) Annual subscription (Individual)

(iv) Annual subscription (Institutional)

(v) Annual subscription (Foreign countries)
- Rs. 300/- per year

- Rs. 1500/-

- Rs. 300/-

- Rs. 500/-

- US \$120/-

Note $\quad$ 1. Please make your cheques or bank drafts in favour of Medical Journal Armed Forces India, payable at Pune. For outstation cheques add Rs. 40/- as bank commission.

2. Intimate non-reciept of issue within three months from the month of publication. 